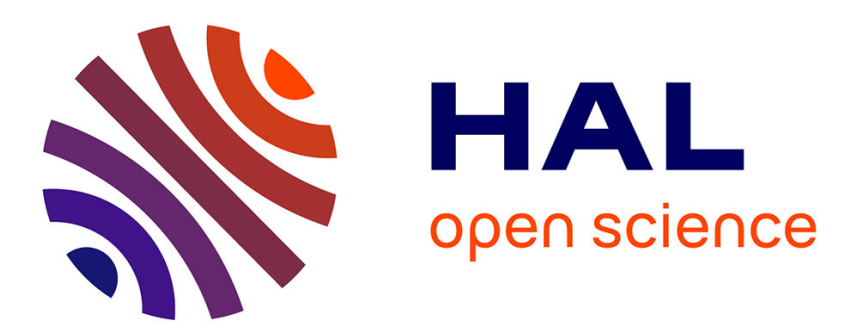

\title{
On planarity of direct product of multipartite complete graphs
}

Laurent Beaudou, Paul Dorbec, Pranava K. Jha, Sylvain Gravier

\section{To cite this version:}

Laurent Beaudou, Paul Dorbec, Pranava K. Jha, Sylvain Gravier. On planarity of direct product of multipartite complete graphs. Discrete Mathematics, Algorithms and Applications, 2009, 1 (1), pp.85-104. 10.1142/S179383090900004X . hal-00387303

\section{HAL Id: hal-00387303 https://hal.science/hal-00387303}

Submitted on 25 May 2009

HAL is a multi-disciplinary open access archive for the deposit and dissemination of scientific research documents, whether they are published or not. The documents may come from teaching and research institutions in France or abroad, or from public or private research centers.
L'archive ouverte pluridisciplinaire HAL, est destinée au dépôt et à la diffusion de documents scientifiques de niveau recherche, publiés ou non, émanant des établissements d'enseignement et de recherche français ou étrangers, des laboratoires publics ou privés. 


\title{
On planarity of direct product of multipartite complete graphs
}

\author{
Laurent Beaudou ${ }^{1}$, Paul Dorbec ${ }^{1}$, Pranava K. Jha ${ }^{2}$ and \\ Sylvain Gravier ${ }^{1}$ \\ ${ }^{1}$ Institut Fourier - ERTé Maths à Modeler \\ 100, rue des Maths \\ 38402 St-Martin d'Hères - FRANCE \\ ${ }^{2}$ Dept. of Computer Science \\ St. Cloud State University \\ St. Cloud, MN 56301 - U.S.A. \\ laurent.beaudou@ujf-grenoble.fr \\ paul.dorbec@ujf-grenoble.fr \\ pkjha@stcloudstate.edu \\ sylvain.gravier@ujf-grenoble.fr
}

\begin{abstract}
The planarity of the direct product of two graphs has been widely studied in the past. Surprisingly, the missing part is the product with $K_{2}$, which seems to be less predictible. In this piece of work, we characterize which subdivisions of multipartite complete graphs, have their direct product with $K_{2}$ planar. This can be seen as a step towards the characterization of all such graphs.
\end{abstract}

Keywords: direct product, planarity, complete graph, multipartite complete graph, subdivision. 


\section{Introduction}

Whether a product of two graphs is planar or non-planar is a naturally-occurring question, having practical relevance. Accordingly, the topic received attention for a long time. For example, Behzad and Mahmoodian [1] presented a complete characterization for the planarity of the Cartesian product, while Jha and Slutzki [5] stated an analogous result with respect to the strong product. The corresponding problem with respect to the direct product (or $\times$-product, which we formally define below) seems to be rather challenging. To that end, Farzan and Waller [3] reported a partial characterization. One case that has not been fully examined in their work is that of the $\times$-product of an arbitrary graph $G$ and $K_{2}$. In this paper, we study the case when $G$ is a multipartite complete graph.

A closely-related topic in this kind of study is that of determining whether or not a graph $G$ is a minor (defined below) of a product of itself with $K_{2}$. The problem is trivial with respect to the Cartesian product and strong product, since $G$ is necessarily a subgraph of each such product. Along these lines, Jha and Slutzki [5] conjectured that every graph $G$ is a minor of $G \times K_{2}$. However, Bottreau and Metivier [2] came up with a counterexample to the conjecture. In particular, they presented a graph $G$, obtainable from $K_{3,3}$ through appropriate edge subdivisions, such that $G$ is non-planar, yet $G \times K_{2}$ is planar (see Figure 1). Interestingly enough, there is another graph $H$, obtainable from $K_{5}$, with a similar property (see Figure 2). Among other things, these counterexamples reinforce the common belief that dealing with the direct product is rather difficult.

When we speak of a graph, we mean a finite, simple and undirected graph. Given two graphs $G$ and $H$, the direct product of these graphs is the graph $G \times H$ on the vertex set $V(G) \times V(H)$, where a vertex $(u, a)$ is adjacent to a vertex $(v, b)$ if and only if $u$ is adjacent to $v$ in $G$ and $a$ to $b$ in $H$. This product is variously known as Kronecker product, tensor product, cardinal product, cross product and categorical product. For details on this product, see [4], and for any undefined terms, see a standard text on graph theory.

A complete $r$-partite graph $K_{p 1, p 2, \ldots, p r}$ is a graph with a vertex set $V=$ $V_{1} \cup V_{2} \cup \ldots \cup V_{r}$ of $p_{1}+p_{2}+\ldots+p_{r}$ vertices, where $V_{i}$ are nonempty disjoint sets, $\left|V_{i}\right|=p_{i}$ for $1 \leq i \leq r$, such that two vertices in $V$ are adjacent if and only if they belong to different $V_{i}$.

The central issue in the structural characterization of a planar graph is that of a graph minor. To that end, an edge-extraction on a graph $G=(V, E)$ consists of removing an edge $e$ resulting in a graph $G-e$, where $V(G-e)=V$ and $E(G-e)=E-\{e\}$. Further, an edge-contraction operation produces a graph from $G$ by removing an edge $\{u, v\}$ of $G$ and identifying (or "merging") $u$ and $v$, thus creating a new single vertex where the latter inherits all of the adjacencies of the merged vertices, without introducing loops or multiple edges.

Definition 1. A graph $H$ is a minor of a graph $G$ if and only if $H$ is obtainable from $G$ by a finite sequence of edge-extraction and edge-contraction operations.

Here is the most useful and most celebrated result on graph planarity.

Theorem 2 (Kuratowski and Wagner $[6,7]$ ). A graph is planar if and only if it has no minor isomorphic to $K_{3,3}$ or $K_{5}$. 

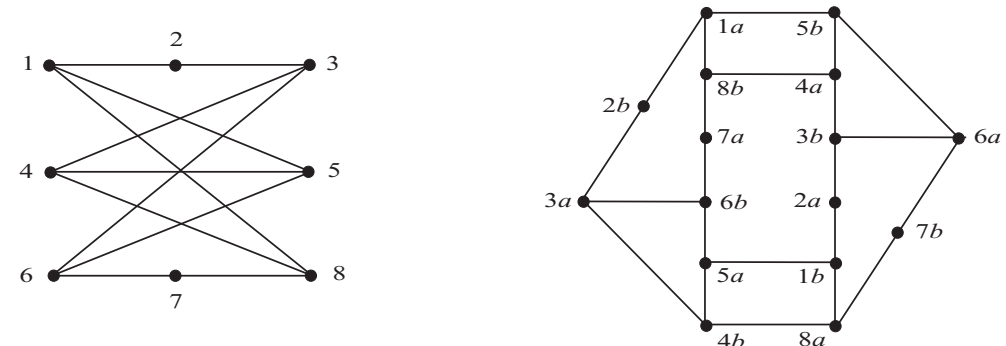

Figure 1: A non-planar graph $G$ and a planar embedding of $G \times K_{2}$
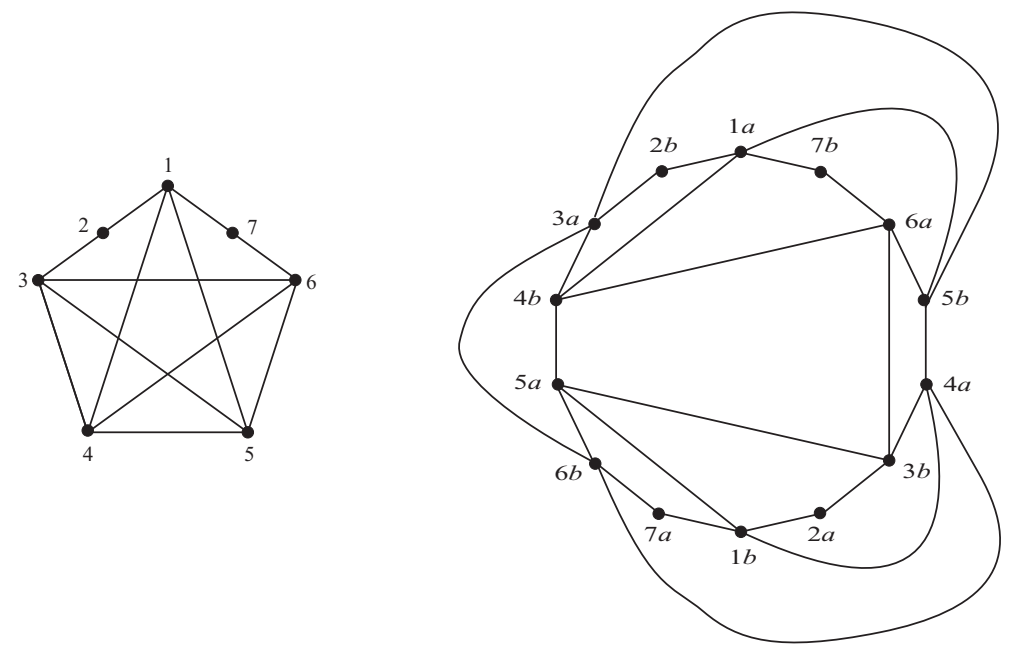

Figure 2: A non-planar graph $H$ and a planar embedding of $H \times K_{2}$ 
Let $G$ be a graph and let $G^{\prime}$ be the graph obtained from $G$ by removing each vertex of degree one. Then $G^{\prime}$ is said to be the 1-contraction of $G$. We now state Farzan and Waller's result on the planarity of the $\times$-product.

Theorem 3. [3]

1. Let $G$ and $H$ be connected graphs with more than four vertices each. Then $G \times H$ is planar if and only if one of the following holds:

(a) one of $G$ and $H$ is a path and the other is 1-contractable to a path or a cycle.

(b) one of $G$ and $H$ is a cycle and the other is 1-contractable to a path.

2. Each of $G \times K_{4}$ and $G \times\left(K_{4}-e\right)$ is planar if and only if $G$ is isomorphic to $K_{2}$.

3. $G \times\left(K_{3}+x\right)$ is planar if and only if $G$ is a path.

4. $G \times K_{1,3}$ is planar if and only if $G$ is a path or a cycle.

5. $G \times C_{4}$ is planar if and only if $G$ is a tree.

6. $G \times C_{3}$ is planar if and only if $G$ is a path or 1-contractable to a path.

\section{Preliminary results}

In the following, we only study the direct product of a graph with $K_{2}$. We denote the vertices of $K_{2}$ by $a$ and $b$, and if $u$ is a vertex of $G$, we denote by $u_{a}$ and $u_{b}$ respectively the vertices $(u, a)$ and $(u, b)$ of $V\left(G \times K_{2}\right)$. In the graph $G \times K_{2}$, there is no edge between two vertices $u_{a}$ and $v_{a}$ (resp. $u_{b}$ and $v_{b}$ ). Therefore, the graph is bipartite, one partition containing all the vertices $u_{a}$, and the other all the vertices $u_{b}$.

Lemma 4. Let $G$ be a graph, and $G^{\prime}$ a graph obtained by subdividing one of its edges twice. Then $G \times K_{2}$ is planar if and only if $G^{\prime} \times K_{2}$ is planar.

Proof. The proof is almost contained in Figure 3. Since $G^{\prime} \times K_{2}$ is obtained from $G \times K_{2}$ by subdividing exactly twice the edges $\left(u_{a}, v_{b}\right)$ and $\left(u_{b}, v_{a}\right)$, if $G \times K_{2}$ is planar, we obtain a natural planar representation of $G^{\prime} \times K_{2}$, and conversely.

Thanks to this lemma, we only have to consider the parity of the number of subdividing vertices for each edge. From now on, we consider subdivisions with 0 or 1 subdividing vertex on each edge of the graph, called 0-1 subdivisions.

Let $G^{\prime}$ be a 0-1 subdivision of a graph $G$. We call a subdividing vertex of $G^{\prime}$ a vertex added to $G$ during a subdivision, the original vertices of $G$ in $G^{\prime}$ being called principal vertices. Further, we call a subdivided edge the path between two principal vertices formed by a subdividing vertex and its two incident edges, by opposition to non-subdivided edges originally in $G$. Two principal vertices in $G^{\prime}$ are said directly linked if there is a non-subdivided edge joining them, and undirectly linked if there is a subdivided edge.

We use the same denominations for the corresponding vertices and edges in $G^{\prime} \times K_{2}$. To understand what underlies all this study, it is important to notice 


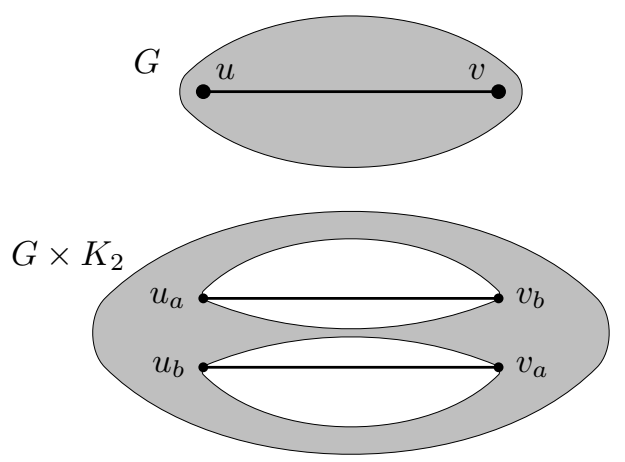

(a) for $G$
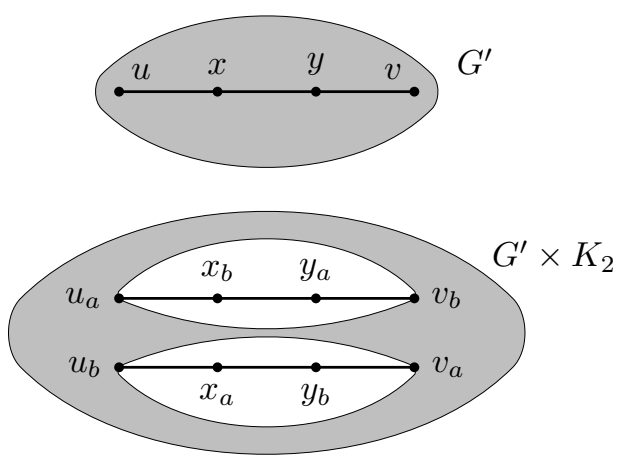

(b) for $G^{\prime}$

Figure 3: A planar representation of $G \times K_{2}$ gives a planar representation of $G^{\prime} \times K_{2}$, and conversely.
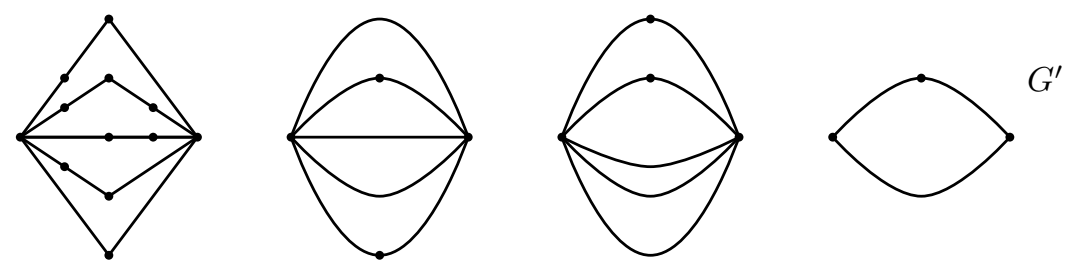

Figure 4: Using Lemma 4 to simplify $K_{1,1,4}$ into a $C_{3}$.

that in $G^{\prime} \times K_{2}$, a non-subdivided edge links two principal vertices $u_{a}$ and $v_{b}$, while a subdivided edge links two principal vertices $u_{a}$ and $v_{a}$ or $u_{b}$ and $v_{b}$ (the subdividing vertex being some $x_{b}$ or $x_{a}$, respectively).

Proposition 5. Let $n \geq 0$ be an integer and $G$ a 0 -1 subdivision of $K_{1, n}, K_{1,1, n}$ or $K_{2, n} . G \times K_{2}$ is a planar graph.

Proof. $K_{1, n}$ is a tree, thus every subdivision of it is a bipartite planar graph. When multiplied by $K_{2}$ we obtain two copies of this planar tree.

Any 0-1 subdivision of $K_{2, n}$ (respectively $K_{1,1, n}$ ) can be seen as two vertices connected by $n$ (resp. $n+1$ ) disjoint paths. Figure 4 shows how by use of Lemma 4, the planarity of $G \times K_{2}$ is equivalent to the planarity of the graph $C_{3} \times K_{2} . C_{3} \times K_{2}$ is the cycle $C_{6}$ on 6 vertices, clearly planar, which proves the proposition.

We now introduce an operation on $0-1$ subdivisions of graphs to simplify the later studies.

Definition 6. Given a 0-1 subdivision $G^{\prime}$ of a graph $G$ and $u$ a principal vertex of $G^{\prime}$, the switch of $G^{\prime}$ around $u$, denoted $S\left(G^{\prime}, u\right)$, is the graph obtained from $G^{\prime}$ by switching the status of every edge incident to u (non-subdivided edges become subdivided and conversely). 


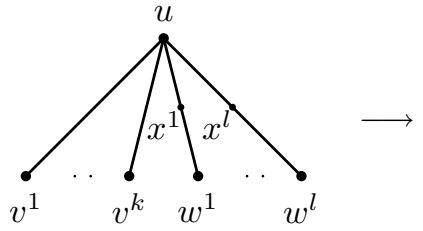

(a) $G$

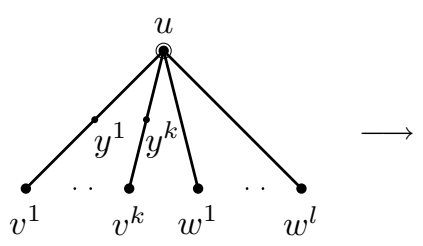

(c) $G^{\prime}$

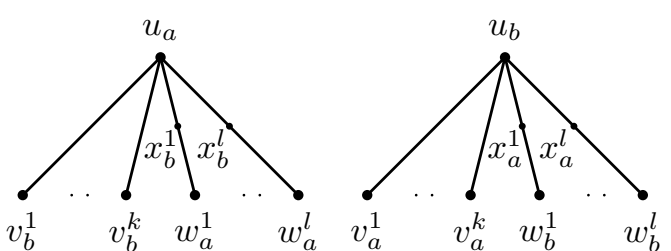

(b) $G \times K_{2}$

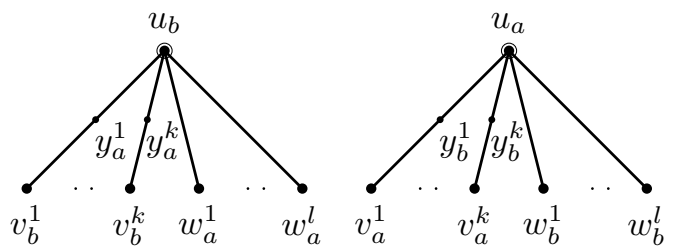

(d) $G^{\prime} \times K_{2}$

Figure 5: Proof of Proposition 7

Proposition 7. Let $G^{\prime}$ be a 0-1 subdivision of some graph $G$. For any principal vertex $u \in G^{\prime}, S\left(G^{\prime}, u\right) \times K_{2}$ is planar if and only if $G^{\prime} \times K_{2}$ is planar.

Proof. Let us denote $v^{1}, \ldots, v^{k}$ principal vertices of $G^{\prime}$ directly linked to $u$ (i.e. by non-subdivided edges), and $w^{1}, \ldots, w^{l}$ principal vertices of $G^{\prime}$ undirectly linked to $u$ (i.e. by subdivided edges). Then in $S\left(G^{\prime}, u\right), u$ is directly linked to $w^{1}, \ldots, w^{l}$ and undirectly linked to $v^{1}, \ldots, v^{k}$. A planar representation of $S\left(G^{\prime}, u\right) \times K_{2}$ can be obtained from $G^{\prime} \times K_{2}$ by interchanging $u_{a}$ and $u_{b}$ as shown in Figure 5 (the subdividing vertices may easily be modified correspondingly). Planarity is clearly conserved.

Two such switches resulting in the identity, the equivalence is proved.

When a $0-1$ subdivision $G^{\prime}$ of a graph $G$ may be obtained by a sequence of switches from another 0-1 subdivision $G^{\dagger}$, we say that the two subdivisions are switch-equivalent. It is straightforward to verify that switch-equivalence is an equivalence relation. Moreover, as a consequence of Proposition 7, we know that if $G^{\prime}$ and $G^{\dagger}$ are switch-equivalent 0-1 subdivisions of a graph $G$, then $G^{\prime}$ is planar if and only if $G^{\dagger}$ is planar.

In the following, we often need to draw some very dense graphs. To avoid very heavy drawings, we use an unusual convention for these drawings: we do not draw the edges, but we draw the subdivided edges with plain lines and the non edges by dashed lines. To sum up, dashed lines represent non existing edges, plain lines represent subdivided edges and invisible lines represent nonsubdivided edges.

The only exception to this rule is Figure 6, where the factor on the left hand side of the drawings use this convention while the product on the right hand side is a usual drawing of a graph.

\section{Dense planar patterns}

In this section, we propose a list of dense graphs that are planar when multiplied by $K_{2}$ (see Figure 6 ). In the proof of the main theorem, we often refer to these 
graphs to prove that some graph is planar.

Lemma 8. All the graphs $A_{1}$ to $A_{7}$ have a planar representation of their direct product with $K_{2}$.

To prove planarity, we propose in Figure 6 a planar representation of each product. The vertex $7_{b}$ is not drawn for $A_{1}, A_{2}$ and $A_{3}$, and some edges are also missing in all the products except for $A_{4}$. These products are symmetric, that is everything inside the external face of our drawings should be copied outside the face, interchanging the ${ }_{a}$ and ${ }_{b} \mathrm{~s}$. Obviously, doing so does not change planarity. The whole graph is drawn for $A_{4}$ since there is no such symmetry.
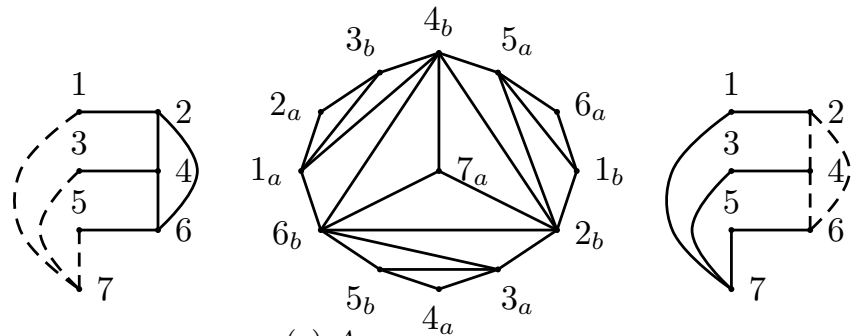

(a) $A_{1}$
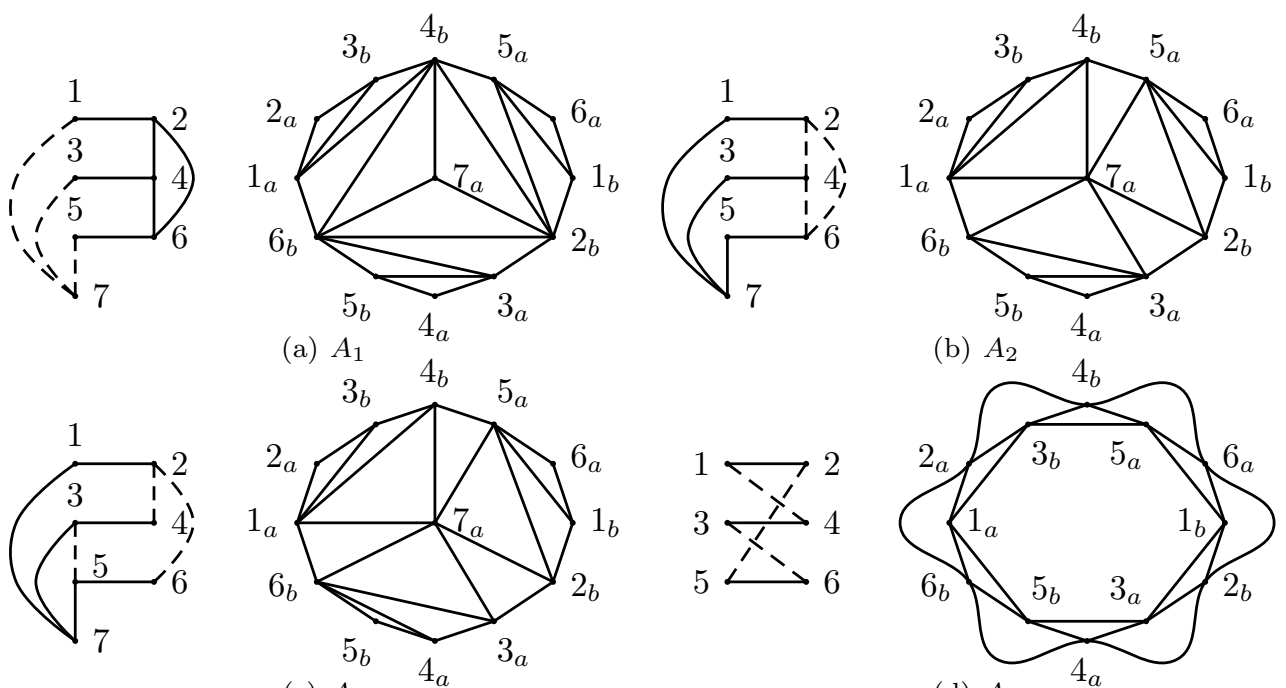

(b) $A_{2}$

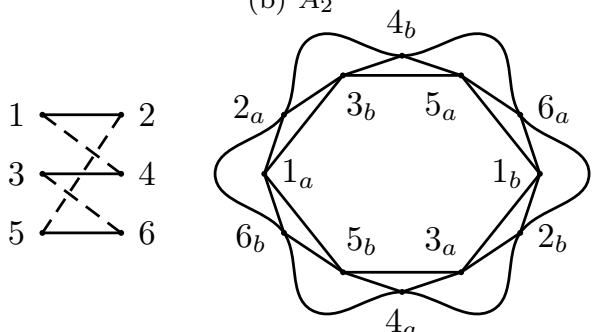

(c) $A_{3}$

(d) $A_{4}$

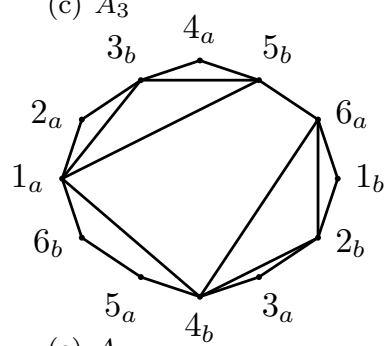

1 '
3
3
5

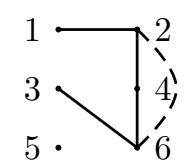

(e) $A_{5}$
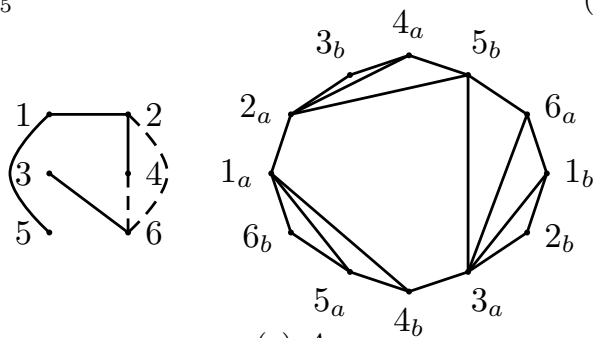

(g) $A_{7}$

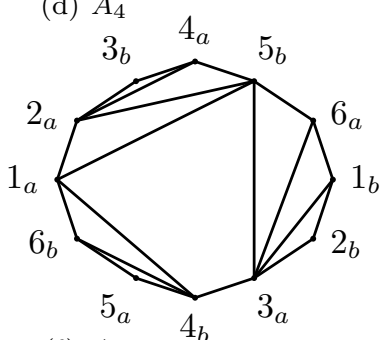

(f) $A_{6}$ 


\section{Non planar patterns}

We propose here a list of some 0-1 subdivisions of graphs that have their product with $K_{2}$ non planar. If one is induced in any 0-1 subdivision of a graph $G$, we can state that $G$ is non planar. To prove non planarity, we just precise the vertices that are contracted in order to get a $K_{5}$ minor or a $K_{3,3}$ minor.

Recall that dashed lines represent non edges, plain lines represent subdivided edges and invisible lines represent non-subdivided edges.

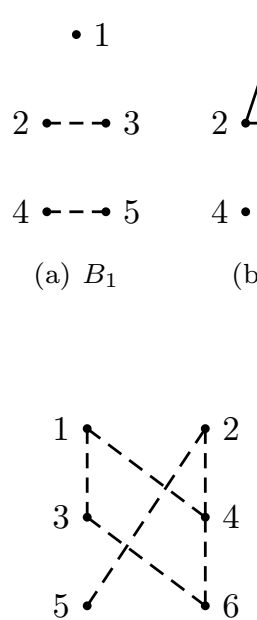

(f) $B_{6}$

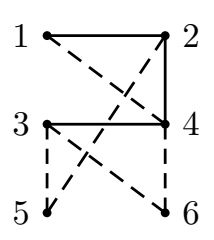

(g) $B_{7}$

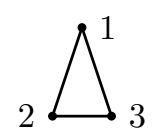

$4 \longmapsto 5$

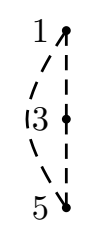

(d) $B_{4}$

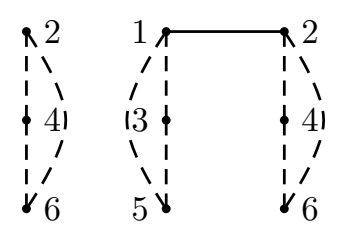

(e) $B_{5}$

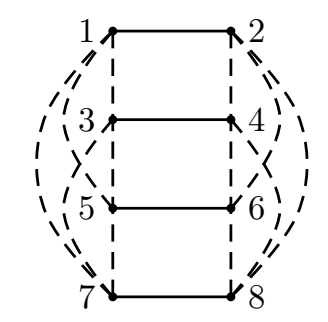

(j) $B_{10}$

Figure 7: Non planar patterns

\section{Proofs}

- $B_{1}:\left(1_{b}\right)\left(5_{b}\right)\left(4_{b}\right)\left[2_{a}\right]\left[3_{a}\right]\left[1_{a}, 2_{b}, 3_{b}, 4_{a}, 5_{a}\right]$ induce a $K_{3,3}$ minor.

- $B_{2}:\left(1_{a}\right)\left(2_{a}\right)\left(3_{a}\right)\left(4_{b}\right)\left(5_{a}, 3_{b}, 4_{a}, 5_{b}\right)$ induce a $K_{5}$ minor.

- $B_{3}$ : it is a bipartite graph so its product with $K_{2}$ is two isomorphic copies of itself. Since $B_{3}$ is not planar, $B_{3} \times K_{2}$ is not planar.

- $B_{4}$ : it is a bipartite graph so its product with $K_{2}$ is two isomorphic copies of itself. Since $B_{4}$ is not planar, $B_{4} \times K_{2}$ is not planar.

- $B_{5}:\left(2_{a}\right)\left(4_{a}\right)\left(6_{a}\right)\left[3_{b}\right]\left[5_{b}\right]\left[1_{a}, 1_{b}, 2_{b}, 3_{a}, 4_{b}, 5_{a}, 6_{b}\right]$ induce a $K_{3,3}$ minor. 
- $B_{6}:\left(1_{a}\right)\left(3_{a}\right)\left(6_{a}\right)\left[2_{b}\right]\left[5_{b}\right]\left[2_{a}, 3_{b}, 5_{a}, 4_{b}, 6_{b}\right]$ induce a $K_{3,3}$ minor.

- $B_{7}:\left(1_{b}\right)\left(2_{b}\right)\left(3_{a}, 4_{a}\right)\left(6_{a}, 5_{b}\right)\left(1_{a}, 2_{a}, 3_{b}, 4_{b}, 6_{b}, 5_{a}\right)$ induce a $K_{5}$ minor.

- $B_{8}:\left(4_{a}, 5_{a}\right)\left(3_{b}, 2_{a}\right)\left(6_{b}, 1_{a}\right)\left(1_{b}, 6_{a}, 5_{b}\right)\left(2_{b}, 3_{a}, 4_{b}\right)$ induce a $K_{5}$ minor.

- $B_{9}:\left(1_{b}\right)\left(5_{b}\right)\left(4_{b}\right)\left[7_{a}\right]\left[4_{a}, 7_{b}, 5_{a}\right]\left[2_{a}, 1_{a}, 6_{b}, 3_{a}, 2_{b}\right]$ induce a $K_{3,3}$ minor.

- $B_{10}:\left(1_{a}, 4_{a}, 5_{b}, 6_{b}\right)\left(1_{a}\right)\left(2_{a}, 3_{b}\right)\left(8_{b}, 7_{b}\right)\left(4_{b}, 5_{a}, 6_{a}\right)$ induce a $K_{5}$ minor.

\section{Main theorem}

Theorem 9. Given $G$ a subdivision of a multipartite complete graph, its product with $K_{2}$ is planar if and only if the corresponding 0-1 subdivision has no partial subgraph switch-equivalent to one of the $B_{i} s$.

To prove this theorem, we enumerate all the multipartite complete graphs and their $0-1$ subdivisions. To reduce the study, we use some arguments related to switches (and Proposition 7) to reduce the number of subdivisions we have to study. Then we represent each of the subdivisions left in a figure with a caption that tells about the planarity of the product of this subdivision with $K_{2}$, as well as its proof. There are three types of captions :

- $\mathrm{P}$ (bipartite) : In that case, since the graph is bipartite, its product with $K_{2}$ consists of two disjoint copies of the graph. Since the graph is also planar, its product is planar.

- $\mathrm{P}\left(A_{i}\right)$ This means the product is planar. The labels on the vertices give a mapping from the subdivision to a subgraph of $A_{i}$.

- $\mathrm{NP}\left(B_{i}\right)$ This caption means the product of this subdivision with $K_{2}$ is non planar. The labels on the vertices give an isomorphism from a partial subgraph of the subdivision to a $B_{i}$.

In the two later cases, sometimes the mapping is not from this $0-1$ subdivision, but from another switch-equivalent subdivision. Whenever so, some vertices are circled with a thin line, they correspond to the vertices we need to switch to get the switch-equivalent subdivision that maps to $A_{i}$ or $B_{i}$.

Subdivisions with less than 4 principal vertices. We prove that the product of subdivisions of $K_{4}$ with $K_{2}$ are planar. Since every principal vertex has 3 neighbours, if a principal vertex $u$ has two or more incident edges subdivided, we can decrease the number of subdivided edges by operating a switch of $G$ around $u$. Thus, we can restrict our study to subdivisions where every principal vertex has at most one subdivided incident edge. Any 0-1 subdivision of $K_{4}$ can be reduced by a sequence of switches to one of the three graphs in Figure 8.

Since all the 0-1 subdivisions of $K_{4}$ are planar when multiplied by $K_{2}$, it is also true for every 0-1 subdivision of any graph of order less or equal than 4 .

Subdivisions with 5 principal vertices. Subdivisions of $K_{1,4}, K_{2,3}$ and $K_{1,1,3}$ are already proved to have a planar product with $K_{2}$ in Proposition 5 . We thus have to study the subdivisions of $K_{1,2,2}, K_{1,1,1,2}$ and $K_{5}$. 


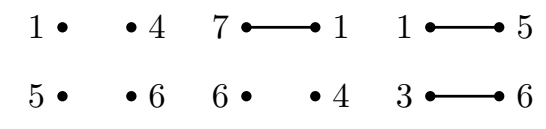
(a) $\mathrm{P}\left(A_{5}\right)$
(b) $\mathrm{P}\left(A_{3}\right)$
(c) $\mathrm{P}\left(A_{7}\right)$

Figure 8: Study of $K_{4}$

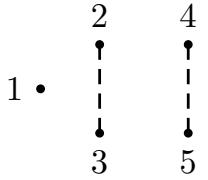

(a) $\mathrm{NP}\left(B_{1}\right)$

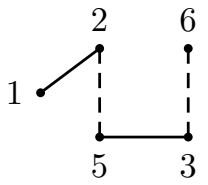

(e) $\mathrm{P}\left(A_{5}\right)$

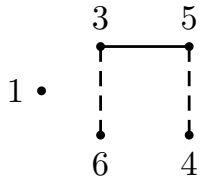

(b) $\mathrm{P}\left(A_{5}\right)$

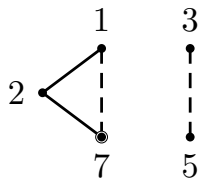

(f) $\mathrm{P}\left(A_{2}\right)$
1 •

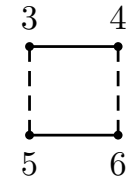

(c) $\mathrm{P}\left(A_{2}\right)$

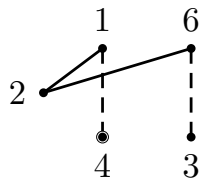

(g) $\mathrm{P}\left(A_{1}\right)$

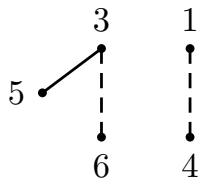

(d) $\mathrm{P}\left(A_{5}\right)$

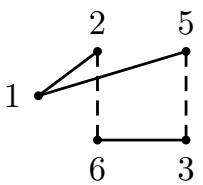

(h) $\mathrm{P}\left(A_{7}\right)$

Figure 9: Study for $K_{1,2,2}$

Let us start with the subdivisions of $K_{1,2,2}$. Any subdivision is switch equivalent to some subdivision whose vertex in the stable set of order 1 has at most two subdivided incident edges and whose other vertices have at most one subdivided incident edge. The corresponding 0-1 subdivisions are listed on Figure 9. Notice that in case (b) for example, the non edge between 4 and 5 is mapped to an edge in $A_{5}$. This is allowed since this a mapping to a partial subgraph of $A_{5}$. Recall that circled vertices (e.g. in (f)) need to be switched before applying the mapping.

Notice also that the subdivision in Figure 9(c) is switch equivalent to the one in Figure 9(g) (switch for example vertices labeled 3 and 6 ) and that the subdivision $9(\mathrm{~h})$ is switch-equivalent to the one in Figure $9(\mathrm{e})$ (switch vertices labeled 1 and 3). Thus, we have the following remark.

Remark 10. All the classes of switch-equivalent $0-1$ subdivisions of $K_{1,2,2}$ are generated by the elements of Figures $9(a), 9(b), 9(d), 9(e), 9(f)$, and $9(g)$.

To treat the subdivisions of $K_{1,1,1,2}$ (see Figure 10(a)), we can consider that the subgraph induced by $a, b$ and $c$ has at most one subdivided edge (otherwise we can switch the vertex with degree 2). Then we can switch vertices $d$ and $e$ so that they have at most one subdivided incident edge. Therefore we only need to check the subdivisions listed in Figure 10. Notice that on case (b) or (c) for example, there is no edge between 2 and 3 in $B_{1}$. Since we look for an isomorphism from a subgraph of the subdivision to $B_{1}$, the subdivision may contain an edge or a subdivided edge linking 2 and 3 .

We now have to study the subdivisions of $K_{5}$. The switching argument allows us to consider only subdivisions with at most two subdivided edges incident to each edge. We obtain the enumeration represented in Figure 11. 


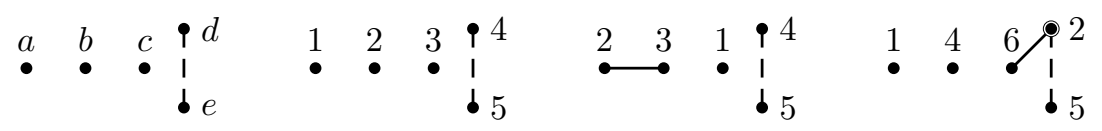
(a) $K_{1,1,1,2}$
(b) NP $\left(B_{1}\right)$
(c) NP $\left(B_{1}\right)$
(d) $\mathrm{P}\left(A_{5}\right)$
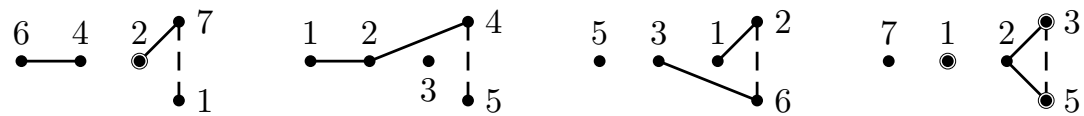
(e) $\mathrm{P}\left(A_{1}\right)$
(f) $\mathrm{P}\left(A_{6}\right)$
(g) $\mathrm{P}\left(A_{6}\right)$
(h) $\mathrm{P}\left(A_{3}\right)$

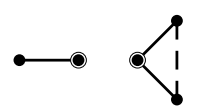

(i) $\mathrm{P}$ (bipartite)

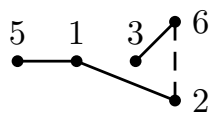

(j) $\mathrm{P}\left(A_{7}\right)$

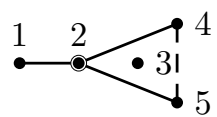

(k) NP $\left(B_{1}\right)$

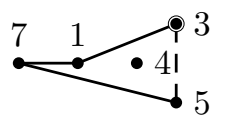

(l) $\mathrm{P}\left(A_{3}\right)$

Figure 10: Study of $K_{1,1,1,2}$
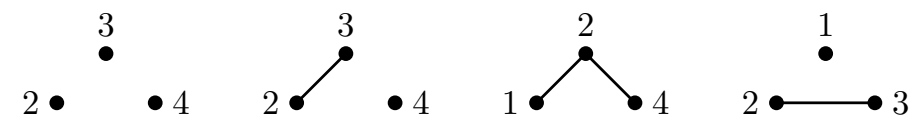

$1 \bullet \bullet 5$
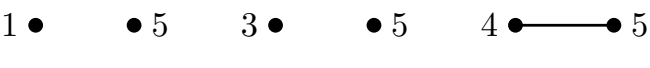

(a) NP $\left(B_{1}\right)$

(b) NP $\left(B_{1}\right)$

(c) $\mathrm{P}\left(A_{6}\right)$

(d) NP $\left(B_{1}\right)$
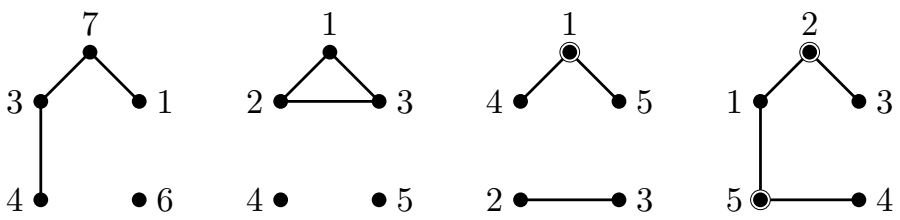

(e) $\mathrm{P}\left(A_{3}\right)$

(f) $\mathrm{NP}\left(B_{2}\right)$

(g) NP $\left(B_{2}\right)$

(h) NP $\left(B_{1}\right)$
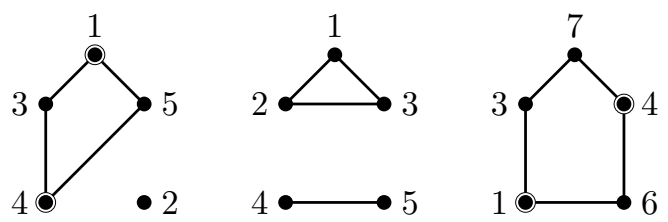
(i) $\mathrm{P}\left(A_{6}\right)$
(j) NP $\left(B_{3}\right)$
(k) $\mathrm{P}\left(A_{3}\right)$

Figure 11: Study of $K_{5}$ 


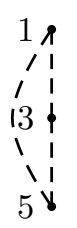

(a) $\mathrm{NP}\left(B_{4}\right)$

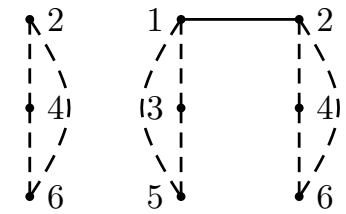

(b) NP $\left(B_{5}\right)$

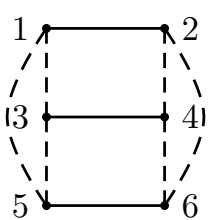

(c) $\mathrm{P}\left(A_{1}\right)$

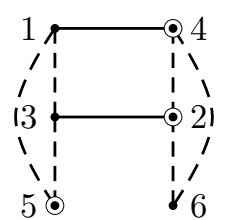

(d) $\mathrm{P}\left(A_{1}\right)$

Figure 12: study of $K_{3,3}$

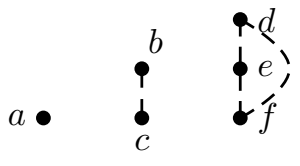

(a) $K_{1,2,3}$

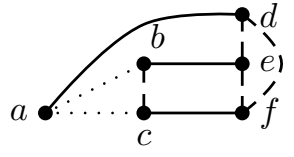

(b)

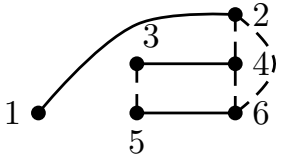

(c) $\mathrm{P}\left(A_{1}\right)$

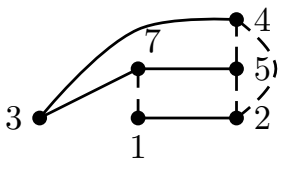

(d) $\mathrm{P}\left(A_{2}\right)$

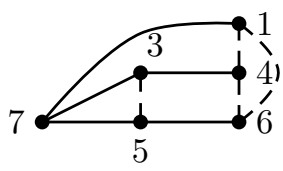

(e) $\mathrm{P}\left(A_{2}\right)$

Figure 13: Study of $K_{1,2,3}$

Subdivisions with 6 principal vertices. Subdivisions of $K_{1,5}, K_{2,4}$ and $K_{1,1,4}$ are already known to have a planar product with $K_{2}$ by Proposition 5. Let us study the $0-1$ subdivisions of $K_{3,3}$. By switch-equivalence, we may consider only subdivisions with at most one subdivided edge incident to each vertex. They are listed in Figure 12.

Since the subdivision in Figure 12(d) is switch-equivalent to the one in Figure 12(c) (with the same switches represented on the figure), the following remark holds.

Remark 11. Given a 0-1 subdivision of $K_{3,3}$, its product with $K_{2}$ is planar if and only if the subdivision is switch-equivalent to the subdivision in Figure 12(c).

Consider now the 0-1 subdivisions of $K_{1,2,3} . K_{1,2,3}$ contains $K_{3,3}$ as a subgraph (see in Figure 13(a), the partition being $\{a, b, c\}$ and $\{d, e, f\}$ ). By the previous remark, we know that whenever the $0-1$ subdivision of this $K_{3,3}$ subgraph is not switch equivalent to the subdivision of Figure 12(c), the graph is not planar. We therefore consider only the other situation, drawn on Figure 13(b), for which we only need to state whether the (dotted) edges $a b$ and $a c$ are subdivided or not. As proved in Figure 13, these subdivisions are all planar.

We now study the $0-1$ subdivisions of $K_{2,2,2}$ (see Figure 14). Consider the induced $K_{1,2,2}$ on the set of vertices $\{a, b, c, d, e\}$. If the $0-1$ subdivision of this subgraph has a non planar product with $K_{2}$ (switch equivalent to Figure 9(a)), then the corresponding subdivision of $K_{2,2,2}$ also has a non planar product with 


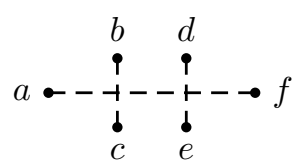

Figure 14: Study for $K_{2,2,2}$

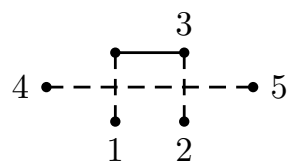

(a) $\mathrm{NP}\left(B_{1}\right)$

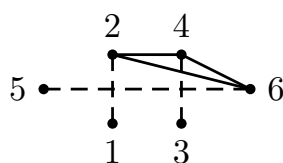

(d) $\mathrm{P}\left(A_{1}\right)$

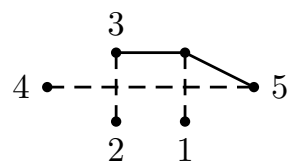

(b) NP $\left(B_{1}\right)$

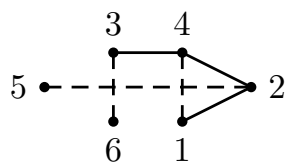

(e) $\mathrm{NP}\left(B_{7}\right)$

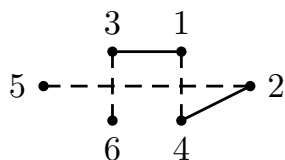

(c) NP $\left(B_{6}\right)$

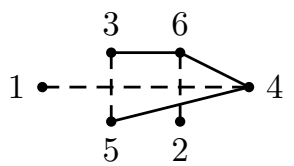

(f) $\mathrm{NP}\left(B_{8}\right)$

Figure 15: Study for 9(b)

$K_{2}$. We therefore study only the cases when this subdivision is planar. By Remark 10, it is then switch equivalent to one of the subdivision in Figures 9(b), 9 (d), $9(\mathrm{e}), 9(\mathrm{f})$, and $9(\mathrm{~g})$. Moreover, we may assume that the remaining vertex $f$ has at most two subdivided incident edges, and if it has two, we can force it to be adjacent to the vertex $d$ by switching around $f$. The remaining subdivision are studied in Figures 15 to 19.

Let us consider $0-1$ subdivisions of $K_{1,1,1,3}$. First remark that $K_{3,3}$ is a subgraph of $K_{1,1,1,3}$ (in Figure 20, consider the sets $\{a, b, c\}$ and $\{d, e, f\}$ ). Therefore, like we did for $K_{2,2,2}$, Remark 11 allows us to consider only subdivisions enumerated in Figure 20.

Let us consider the 0-1 subdivisions of $K_{1,1,2,2}$ that are planar when multiplied by $K_{2}$. As it contains a $K_{3,3}$ as a partial subgraph, there is a sequence of switches leading to one of the graphs in Figures 21(a) and 21(b).

We now consider $0-1$ subdivisions of $K_{1,1,1,1,2}$. They are necessarily obtained from a good subdivision of $K_{1,1,2,2}$ (Figures 22(c), 23(c),23(d)), by adding an edge either subdivided or not. So the enumeration in Figure 24 is complete.

Let us continue with 0-1 subdivisions of $K_{6}$. It contains a subdivision of $K_{1,1,1,1,2}$ which must be planar when multiplied by $K_{2}$. Thus, there exists a sequence of switches resulting in one of the subgraphs in Figures 24(a) 24(b). We just need to decide the status of the deleted edge (see Figure 25).

Graphs with 7 principal vertices. Subdivisions of $K_{1,6}, K_{2,5}$ and $K_{1,1,5}$ are already known to be planar by Proposition 5. Let us study the 0-1 subdivisions of $K_{3,4}$. We can consider the induced $K_{3,3}$ in order to enumerate these graphs. Moreover, the fourth vertex of the 4 -stable can be considered to have at most one incident subdivided edge (see Figure 26).

Let us study 0-1 subdivision of $K_{1,2,4}$. The induced subdivision of $K_{3,4}$ can be reduced to the graph in Figure 26(a) by a sequence of switches. These graphs are studied in Figure 27

In order to study subdivisions of $K_{1,3,3}$ we consider the induced $K_{3,3}$ and 


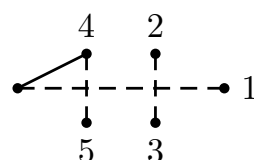

(a) NP $\left(B_{1}\right)$

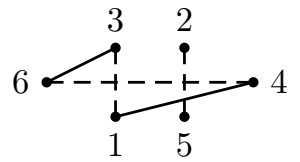

(d) NP $\left(B_{6}\right)$

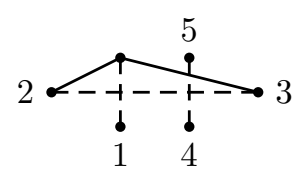

(b) NP $\left(B_{1}\right)$

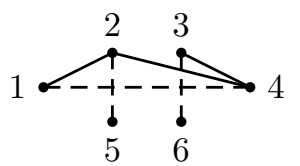

(e) NP $\left(B_{7}\right)$

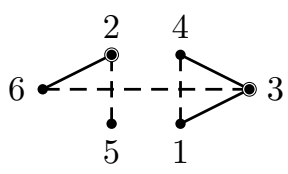

(g) $\mathrm{P}\left(A_{5}\right)$

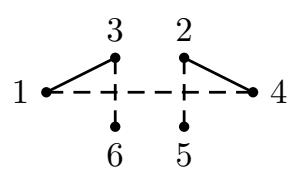

(c) NP $\left(B_{6}\right)$

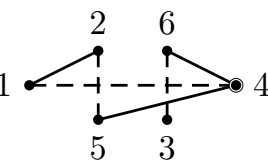

(f) $\mathrm{NP}\left(B_{7}\right)$

Figure 16: Study for 9(d)

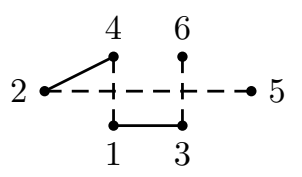

(a) NP $\left(B_{6}\right)$

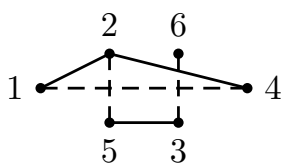

(d) $\mathrm{P}\left(A_{5}\right)$

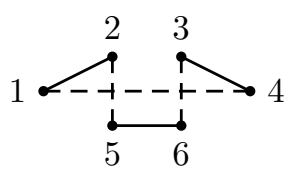

(b) $\mathrm{P}\left(A_{4}\right)$

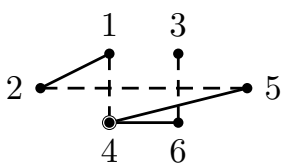

(e) NP $\left(B_{7}\right)$

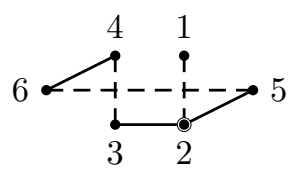

(c) $\mathrm{P}\left(A_{1}\right)$

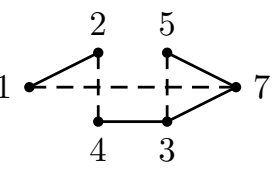

(f) $\mathrm{P}\left(A_{3}\right)$

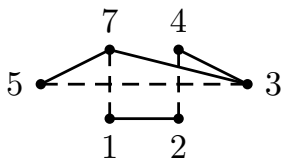

(g) $\mathrm{P}\left(A_{3}\right)$

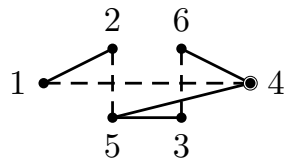

(h) NP $\left(B_{7}\right)$

Figure 17: Study for 9(e)

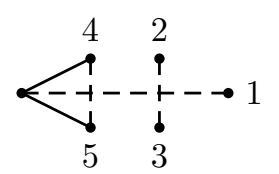

(a) NP $\left(B_{1}\right)$

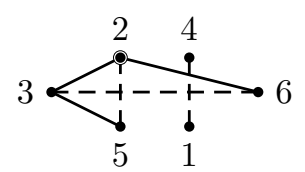

(b) $\mathrm{P}\left(A_{5}\right)$

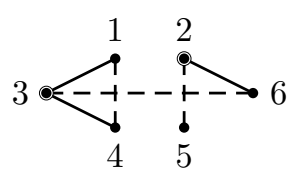

(c) $\mathrm{P}\left(A_{5}\right)$

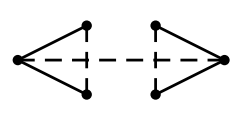

(d) P (planar and bipartite)

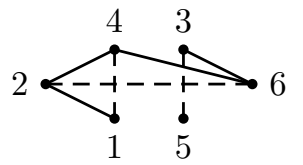

(e) $\mathrm{P}\left(A_{6}\right)$

Figure 18: Study for 9(f) 


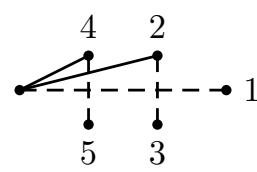

(a) NP $\left(B_{1}\right)$

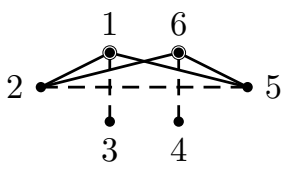

(d) NP $\left(B_{6}\right)$

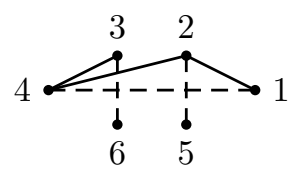

(b) $\mathrm{NP}\left(B_{7}\right)$

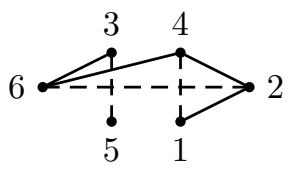

(e) $\mathrm{P}\left(A_{6}\right)$

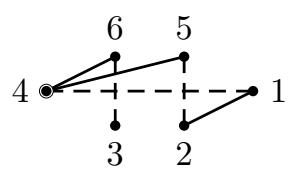

(c) NP $\left(B_{7}\right)$

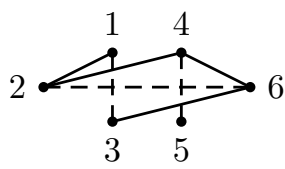

(f) $\mathrm{P}\left(A_{6}\right)$

Figure 19: Study for $9(\mathrm{~g})$

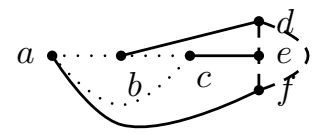

(a) Unknown edges

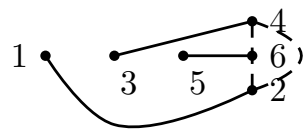

(b) $\mathrm{P}\left(A_{2}\right)$

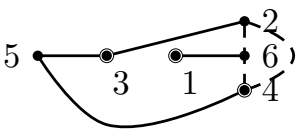

(c) $\mathrm{P}\left(A_{7}\right)$

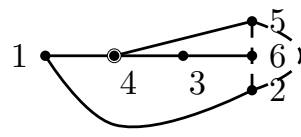

(d) $\mathrm{P}\left(A_{6}\right)$

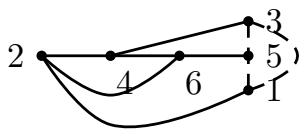

(e) $\mathrm{P}\left(A_{1}\right)$

Figure 20: Study of $K_{1,1,1,3}$

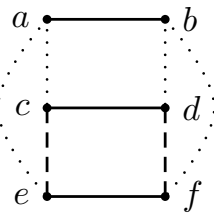

(a)

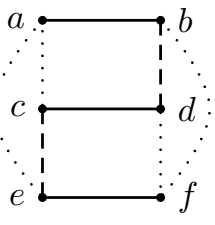

(b)

Figure 21: $K_{1,1,2,2}$ contains a $K_{3,3}$ 

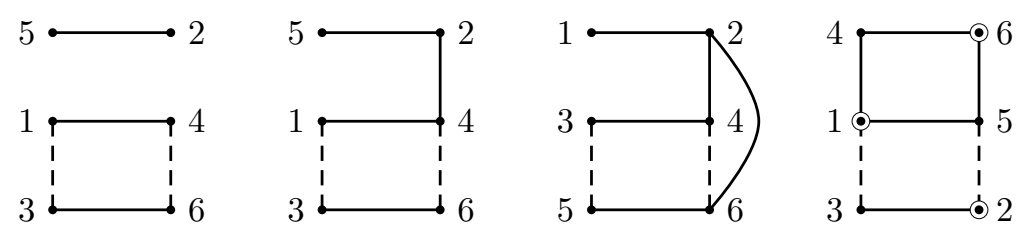

(a) $\mathrm{NP}\left(B_{6}\right)$

(b) $\mathrm{NP}\left(B_{6}\right)$

(c) $\mathrm{P}\left(A_{1}\right)$

(d) NP $\left(B_{6}\right)$

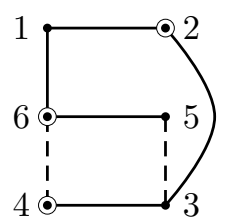

(e) NP $\left(B_{8}\right)$

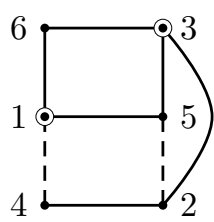

(f) $\mathrm{NP}\left(B_{7}\right)$

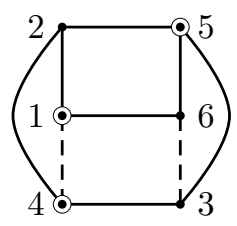

(g) NP $\left(B_{6}\right)$

Figure 22: Study of 21(a)

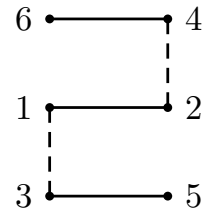

(a) $\mathrm{NP}\left(B_{5}\right)$

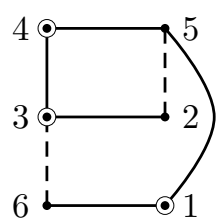

(e) $\mathrm{NP}\left(B_{7}\right)$

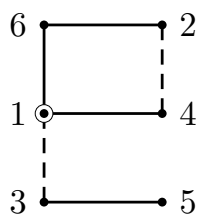

(b) NP $\left(B_{5}\right)$

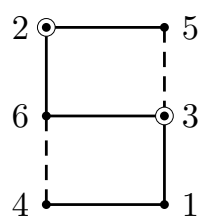

(f) $\mathrm{NP}\left(B_{7}\right)$

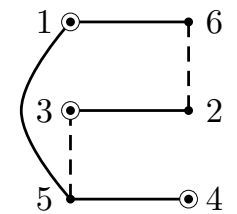

(c) $\mathrm{P}\left(A_{6}\right)$

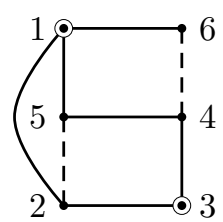

(g) NP $\left(B_{8}\right)$

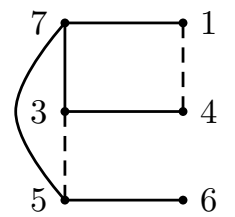

(d) $\mathrm{P}\left(A_{3}\right)$

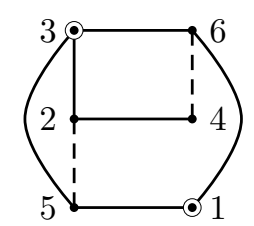

(h) $\mathrm{NP}\left(B_{7}\right)$

Figure 23: Study of 21(b) 


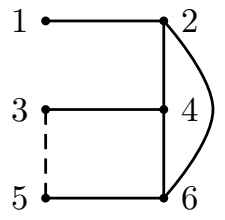

(a) $\mathrm{P}\left(A_{1}\right)$

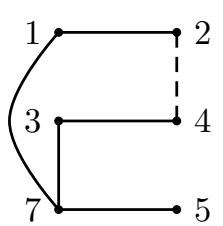

(e) $\mathrm{P}\left(A_{2}\right)$

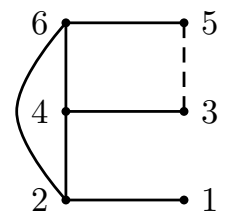

(i) $\mathrm{P}\left(A_{1}\right)$

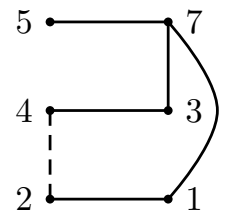

(b) $\mathrm{P}\left(A_{2}\right)$

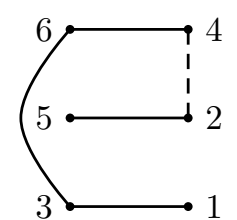

(f) NP $\left(B_{6}\right)$

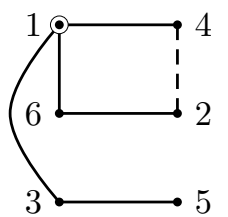

(j) NP $\left(B_{5}\right)$

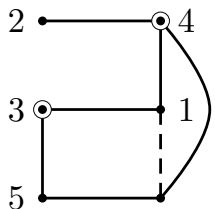

(c) NP $\left(B_{1}\right)$

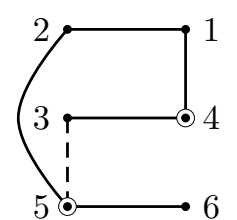

(g) NP $\left(B_{5}\right)$

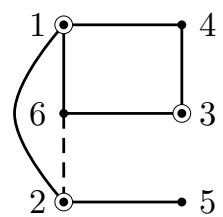

(k) NP $\left(B_{5}\right)$

Figure 24: Study of $K_{1,1,1,1,2}$

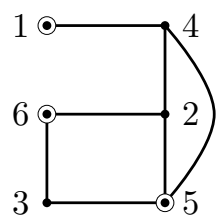

(a) $\mathrm{NP}\left(B_{5}\right)$

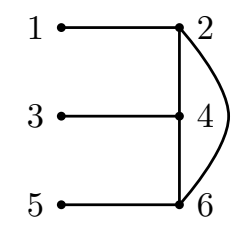

(b) $\mathrm{P}\left(A_{1}\right)$

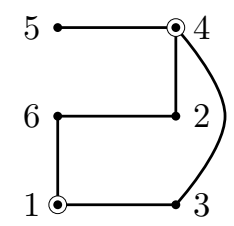

(c) NP $\left(B_{5}\right)$

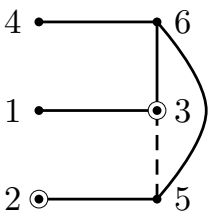

(d) $\mathrm{P}\left(A_{1}\right)$

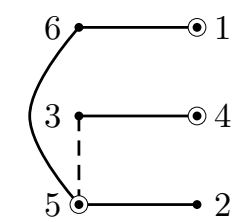

(h) NP $\left(B_{5}\right)$

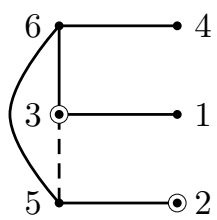

(l) $\mathrm{P}\left(A_{1}\right)$

Figure 25: Study of $K_{6}$

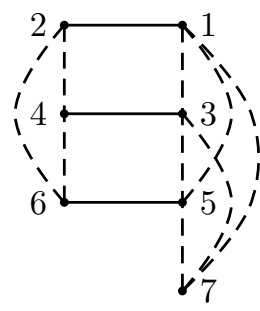

(a) $\mathrm{P}\left(A_{1}\right)$

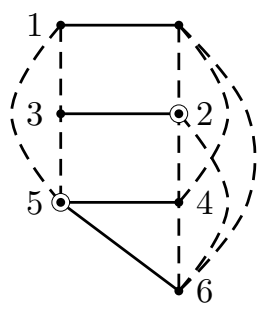

(b) NP $\left(B_{5}\right)$

Figure 26: Study of $K_{3,4}$ 


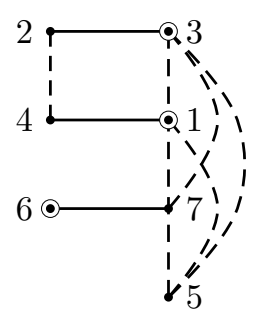

(a) $\mathrm{P}\left(A_{1}\right)$

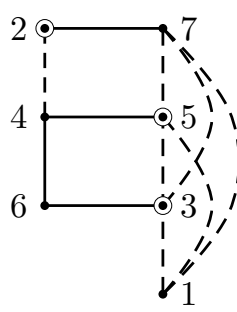

(b) $\mathrm{P}\left(A_{1}\right)$

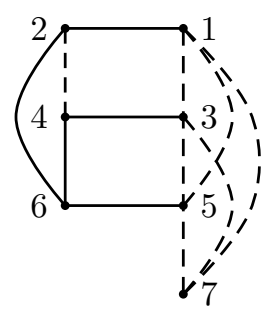

(c) $\mathrm{P}\left(A_{1}\right)$

Figure 27: Study of $K_{1,2,4}$

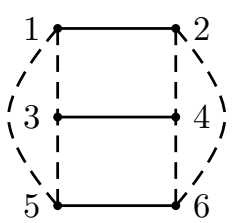

$\cdot 7$

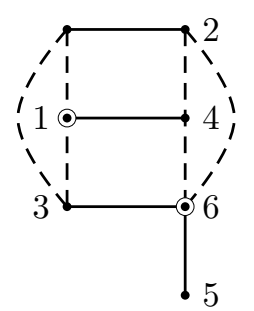

(b) NP $\left(B_{5}\right)$

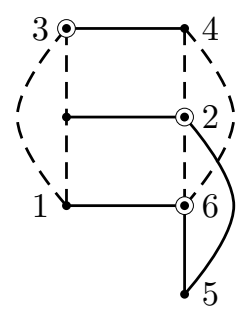

(c) $\mathrm{NP}\left(B_{5}\right)$

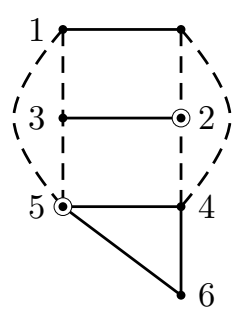

(d) NP $\left(B_{5}\right)$

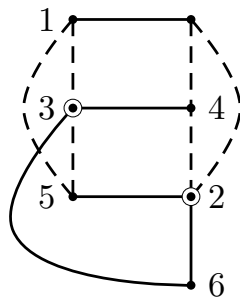

(e) NP $\left(B_{5}\right)$

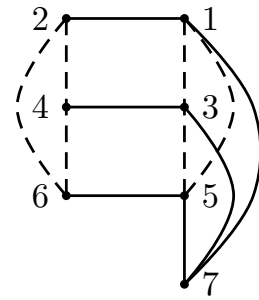

(f) $\mathrm{P}\left(A_{3}\right)$

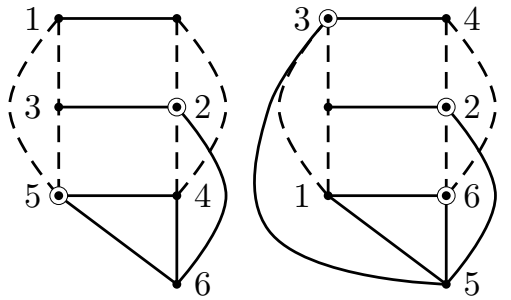

(h) NP $\left(B_{5}\right)$

Figure 28: Study of $K_{1,3,3}$

assume that the remaining vertex has at most 3 incident subdivided edges. The resulting enumeration is in Figure 28.

To study subdivisions of $K_{2,2,3}$, we consider the included subdivision of $K_{3,4}$, we get the graph drawn in Figure 29(a). it appears that by adding setting 0 or 1 unknown edge to subdivided, we get a $B_{9}$ as a subgraph, so that it is not planar. The only way to ensure that the graph does not contain $B_{9}$ is to set at least two edges as subdivided. And if there are exactly two of them, they must be distinct from $d f$ and $b f$ (by symmetry) or it would also contain a $B_{9}$. Therefore, the enumeration in Figure 29 is complete.

To study $K_{1,1,1,4}$ we consider the $K_{3,4}$ contained (see Figure 26 ).

Let us study subdivisions of $K_{1,1,2,3}$. based on the only planar configuration for $K_{2,2,3}$ which can be placed in two different ways in this graph we get the enumeration in Figure 31.

For the study of $K_{1,2,2,2}$ we consider its partial subdivision isomorphic to a subdivision of $K_{2,2,3}$. We conclude that there is a sequence of switches leading 


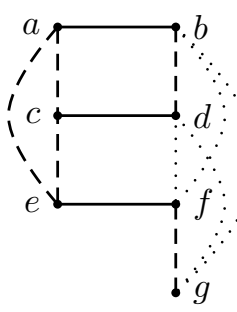

(a)

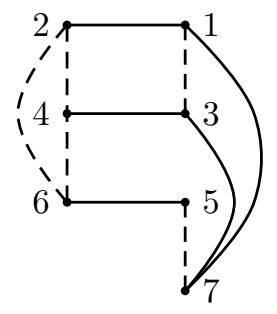

(b) $\mathrm{P}\left(A_{2}\right)$

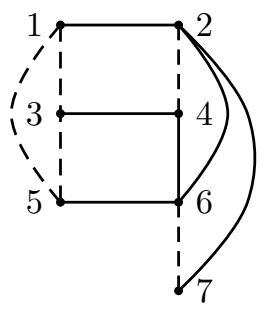

(c) NP $\left(B_{9}\right)$

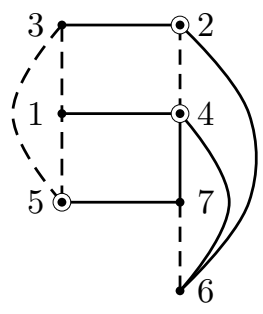

(d) NP $\left(B_{9}\right)$

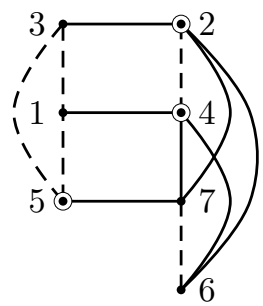

(e) NP $\left(B_{9}\right)$

Figure 29: Study of $K_{2,2,3}$

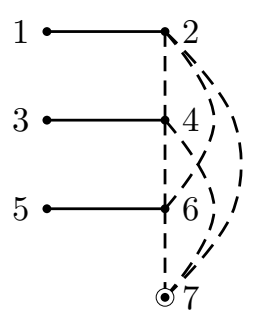

(a) $\mathrm{P}\left(A_{2}\right)$

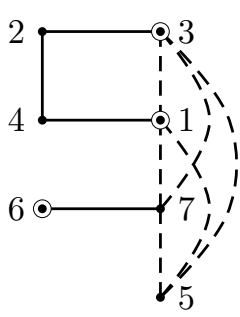

(b) $\mathrm{P}\left(A_{1}\right)$

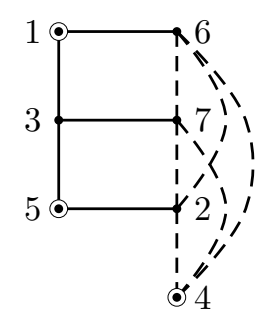

(c) $\mathrm{P}\left(A_{2}\right)$

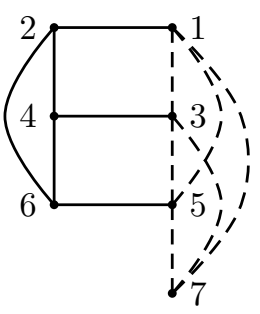

(d) $\mathrm{P}\left(A_{1}\right)$

Figure 30: Study of $K_{1,1,1,4}$
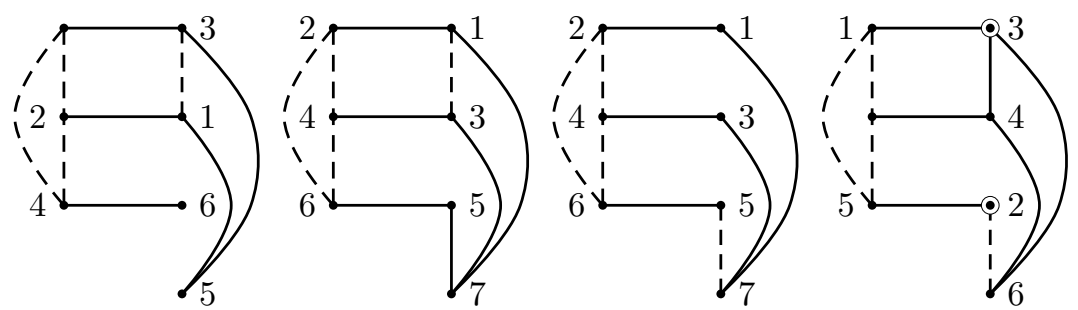

(a) $\mathrm{NP}\left(B_{5}\right)$

(b) $\mathrm{P}\left(A_{2}\right)$

(c) $\mathrm{P}\left(A_{2}\right)$

(d) $\mathrm{NP}\left(B_{5}\right)$

Figure 31: Study of $K_{1,1,2,3}$ 


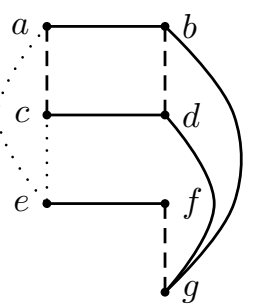

(a)

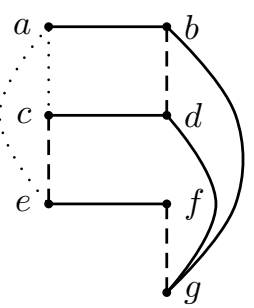

(e)

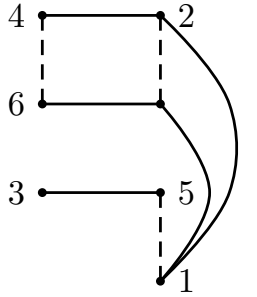

(b) NP $\left(B_{5}\right)$

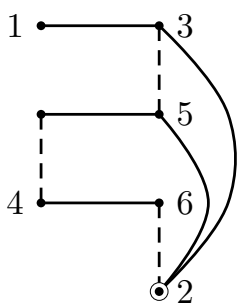

(f) NP $\left(B_{5}\right)$

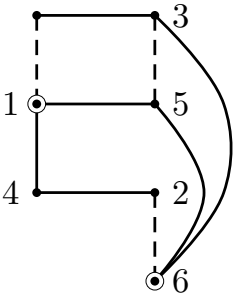

(c) NP $\left(B_{5}\right)$

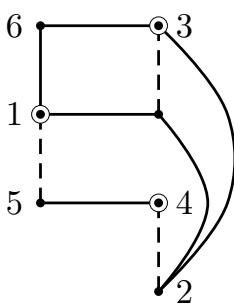

(g) NP $\left(B_{5}\right)$

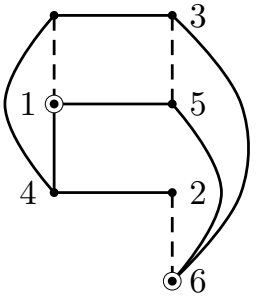

(d) NP $\left(B_{5}\right)$

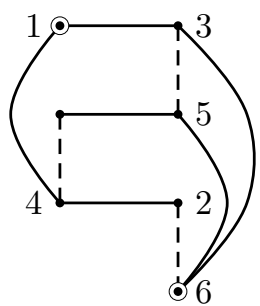

(h) NP $\left(B_{5}\right)$

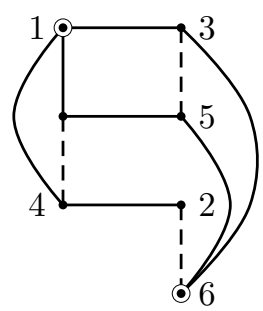

(i) NP $\left(B_{5}\right)$

Figure 32: Study of $K_{1,2,2,2}$

to configurations in Figures 32(a) or 32(e). Thus the enumeration is complete and we can conclude that there is no subdivision of $K_{1,2,2,2}$ resulting in planar graph when multiplied by $K_{2}$.

For $K_{1,1,1,1,3}$, we start from planar configurations of $K_{1,1,2,3}$ (see Figures $31(\mathrm{~b})$ and $31(\mathrm{c})$ ). So that we only have to study three configurations (see Figure $33)$.

Any subdivision of $K_{1,1,1,2,2}$ contains a subdivision of $K_{1,2,2,2}$ and thus is a non planar configuration. The same stands for $K_{1,1,1,1,1,2}$ and $K_{7}$.

Graphs with 8 principal vertices. Subdivisions of $K_{1,7}, K_{2,6}$ and $K_{1,1,6}$ are already known to be planar by Proposition 5 . Let us study the $0-1$ subdivisions of $K_{3,5}$. We can consider the induced $K_{3,4}$ in order to enumerate these graphs. Moreover, the fifth vertex of the 5 -stable can be considered to have at most one incident subdivided edge (Figure 34 ).

We now check that any subdivisions of $K_{4,4}$ is non planar when multiplied by $K_{2}$. We consider the subdivision of $K_{3,4}$ included and check that the remaining vertex can be considered to have at most two incident subdivided edges.

Other multipartite complete graphs with 8 vertices contain either a $K_{3,5}$ 


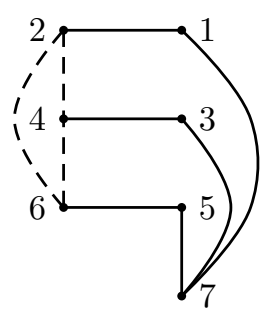

(a) $\mathrm{P}\left(A_{2}\right)$

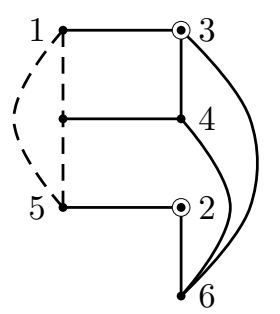

(b) NP $\left(B_{5}\right)$

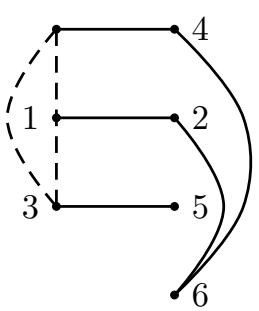

(c) NP $\left(B_{5}\right)$

Figure 33: Study of $K_{1,1,1,1,3}$

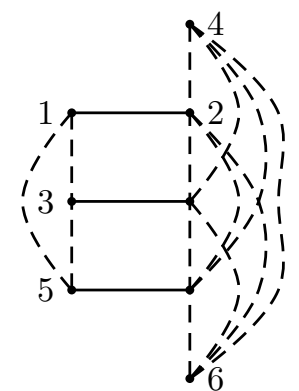

(a) $\mathrm{NP}\left(B_{5}\right)$

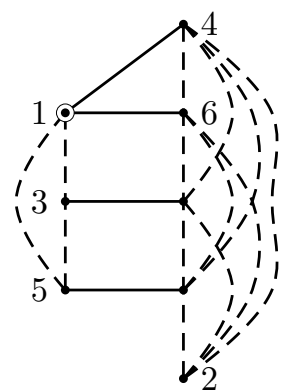

(b) $\mathrm{NP}\left(B_{5}\right)$

Figure 34: Study of $K_{3,5}$

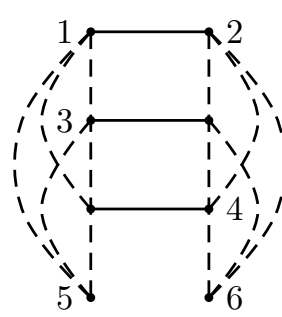

(a) NP $\left(B_{5}\right)$

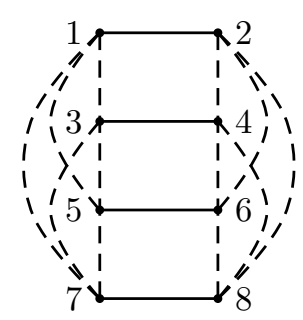

(b) NP $\left(B_{10}\right)$

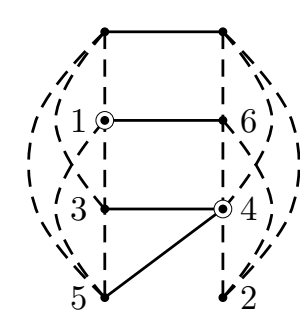

(c) $\mathrm{NP}\left(B_{5}\right)$

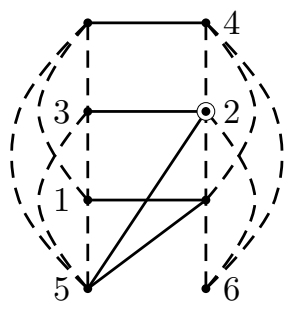

(e) NP $\left(B_{5}\right)$

Figure 35: Study of $K_{4,4}$ 
or a $K_{4,4}$ and thus can never be planar when multiplied by $K_{2}$. We observe that the only multipartite complete graphs with 8 vertices that admit a planar configuration are $K_{1,7}, K_{2,6}$ and $K_{1,1,6}$.

Graphs with $n \geq 9$ principal vertices. Subdivisions of $K_{1, n-1}, K_{2, n-2}$ and $K_{1,1, n-2}$ are already known to be planar by Proposition 5. Let us consider a 0-1 subdivision of $K_{n_{1}, n_{2} \ldots n_{k}}\left(k \geq 2\right.$ since $K_{n}$ contain $\left.K_{8}\right)$. We assume that the $n_{i}$ 's are listed from smallest to biggest. If there exists a subset of $S \subset\{1 \ldots n\}$ such that $\sum_{i \in S} n_{i}=3$ or 4 . Then it is clearly non planar since $n \geq 9$ and thus the graph contains a $K_{3,5}$, or a $K_{4,4}$.

Therefore, we can consider there is no such subset. The only way is to have either $n_{1} \geq 5$, or $n_{1}=2$ and $n_{2} \geq 5$, or $n_{1}=n_{2}=1$ and $n_{3} \geq 5$.

If $n_{1} \geq 5$, then $n_{2} \geq n_{1} \geq 5$ and thus the subdivisions contains a $K_{3,5}$ and is non planar when multiplied by $K_{2}$.

If $n_{1}=2$ and $n_{2} \geq 5$, since we suppose it is no $K_{2, n-2}$, we can assume that $k \geq 3$. Thus, $n_{3} \geq n_{2} \geq 5$. We can exhibit a $K_{3,5}$ and the configuration is non planar.

Similarly, if $n_{1}=n_{2}=1$ and $n_{3} \geq 5$, we can assume that $k \geq 4\left(K_{1,1, n-2}\right.$ already studied). Thus, $n_{3} \geq n_{2} \geq 5$. We can exhibit a $K_{3,5}$ and the configuration is non planar.

\section{References}

[1] M. Behzad and S.E. Mahmoodian, On topological invariants of products of graphs, Canad. Math. Bull., 12(1969), 157-166.

[2] A. Bottreau and Y. Métivier, Some remarks on the Kronecker product of graphs, Inf. Process. Lett., 68(1998), 55-61.

[3] M. Farzan and D.A. Waller, Kronecker products and local joins of graphs, Canad. J. Math., 29(1977), 255-269.

[4] W. Imrich and S. Klavžar, Product Graphs: Structure and Recognition, J. Wiley \& Sons, New York, 2000.

[5] P.K. Jha and G. Slutzki, A note on outerplanarity of product graphs, Zastos. Mat. (Applic. Math.), 21(1993), 537-544.

[6] K. Kuratowski, Sur le problème des courbes gauches en topologie, Fund. Math., 15(1930), 271-283.

[7] K. Wagner, Über eine Erweiterung eines Satzes von Kuratowski, Duet. Math., 2(1937), 280-285. 